\title{
Optimization of Dose Parameters to Patients Undergoing CT Scan Using Four Different CT Scanners with International Guidelines
}

\author{
Issahaku Shirazu $^{1,4}$, Y. B Mensah ${ }^{2}$, Cyril Schandorf ${ }^{3}$, S. Y. Mensah ${ }^{1}$, Alfred Owusu ${ }^{1}$ \\ ${ }^{1}$ University of Cape Coast, School of Agriculture and Physical Sciences Faculty of Physical Sciences, Department of Physics, \\ Cape coast, Ghana \\ ${ }^{2}$ University of Ghana Medical School, Department of Radiology, Korle-Bu Teaching Hospital, Accra Ghana \\ ${ }^{3}$ Graduate School of Nuclear and Allied Sciences, University of Ghana, Legon, Ghana \\ ${ }^{4}$ Ghana Atomic Energy Commission, Radiological and Medical Sciences Research Institute, Medical Radiation Physics Centre, \\ Accra, Ghana
}

\begin{abstract}
Although the use of CT in medical diagnosis delivers radiation doses to patients that are higher than those from other radiological procedures, lack of proper optimized protocols could be an additional source of increased dose in developing countries. The aims of this study is to determine the variations of dose to patients undergoing CT scan using four different CT scanners with different CT scanning protocols for the purposes of optimizations and to compare with other available international standard and guidelines. The method involve the use of patients scanning protocol and image data to estimate patient body regional doses with four common CT examinations. These were obtained from four $\mathrm{CT}$ units/hospitals in Ghana. A large variation of mean body regional doses among different CT scanners were observed for similar CT examinations. These variations largely originated from different CT scanning protocols used, with different CT scanner type. The measured CTDI $\mathrm{VOL}_{\mathrm{L}}$ with GE Lightspeed VCT 64 scanner for head, chest, abdomen and pelvis were 7.7mGy, $12.5 \mathrm{mGy}, 14.4 \mathrm{mGy}$, and $12.9 \mathrm{mGy}$, respectively. Similarly, Philip 16 scanner recorded $6.6 \mathrm{mGy}, 13.1 \mathrm{mGy}, 14.8 \mathrm{mGy}$, and $14.5 \mathrm{mGy}$ respectively. Furthermore, Siemen Emotion 16 scanner recorded $5.9 \mathrm{mGy}, 14.2 \mathrm{mGy}, 16.8 \mathrm{mGy}$ and $12.0 \mathrm{mGy}$ respectively. While, Toshiba Aquilion one scanner had CTDIVOL value which varies as $7.2 \mathrm{mGy}, 13.4 \mathrm{mGy}, 15.2 \mathrm{mGy}$ and $13.5 \mathrm{mGy}$ respectively. In conclusion the values were mostly lower than the values of CTDI and DLP as reported in literature for EC, IAEA, ICRP, ACR and AAPM Guidelines.
\end{abstract}

Keywords: Optimisation, Scanning Protocol, Computer Tomography, Dose Reference Level

\section{INTRODUCTION}

Most regulatory bodies have made the use of diagnostic reference levels mandatory. This has been endorsed by all professional and international organizations, including the International Commission on Radiological Protection (ICRP), American College of Radiology (ACR), American Association of Physicists in Medicine (AAPM), United Kingdom Health Protection Agency, International Atomic Energy Agency (IAEA), and European Commission (EC) [1]. Generally, reference levels are typically set at the $75^{\text {th }}$ percentile of the dose distribution from a survey conducted across a broad user base (i.e., large and small facilities, public and private, hospital and out-patient) using a specified dose measurement protocol [1]. They are established by both regionally and nationally bodies and considerable variations have been seen across both regions and countries. It is encouraged that dose surveys should be repeated periodically to establish new reference levels, which can demonstrate changes in both the mean and standard deviation of the dose distribution [2].

In fact patient doses from CT procedures are relatively higher than doses from other ionizing radiation based imaging modalities. For example, one CT examination of the chest delivers about 400 times the dose delivered by a conventional chest X-ray examination [3]. Even though CT represents only $5 \%$ of the total number of medical X-ray procedures worldwide, this high dose procedure contributes about $34 \%$ of the annual 
collective dose from all medical X-ray examinations to the world population [3]. This contribution is inevitable because it results from a combination of high dose per examination per frequent use of $\mathrm{CT}$ examination in diagnosis [3]. Ghana is not an exception to this trend since its inception, where the use of CT for medical examination started in the early 90s. Currently, there are about 32 CT scanners in the country, and nearly 210,000 CT examinations are performed annually. With an average of $20 \mathrm{CT}$ examination per center per day. This constitutes about $20 \%$ of all medical X-ray procedures performed in Ghana. Indeed, the increased use of this high dose procedure has been of great concern globally because of the high possibility of induced undesired health effects, such as induction of cancer, in patients [4].

The most useful way to assess regional body doses is by the use of DLP with the region-specific normalizing constant $\left(\mathrm{E}_{\mathrm{DLP}}\right)$. DLP is the product of CTDI and the length of the area been scan [5]. The CT dose index (CTDI) is a standardized measure of radiation dose output of a CT scanner. This allows the user to compare radiation output of different $\mathrm{CT}$ scanners. In the past $\mathrm{CTDI}_{100}$ (measured over a $100 \mathrm{~mm}$ long ionization chamber) and CTDIw (weighted average of dose across a single slice) were used, however for helical scanners in current use, the parameter CTDIvol is used [6].

\section{A. Objective}

The aim of this study is to determine the CTDI and the DLP values of patients undergoing CT examination in Ghana and to compare the values with other international standards and guidelines, this is intended to assess the effect of CT scanning protocols on patient doses.

Furthermore, it is to determine the variations of effective dose to patients undergoing CT scan with similar scanning protocols for the purposes of dose optimizations.

\section{B. Literature Review}

In clinical practice, there are a number of ways to measures radiation dose that radiological clinicians commonly used when addressing the biological effects on human body during CT examination. Some of these measured quantities include: Absorbed dose, Organ dose, Dose Equivalent, effective dose and Dose Rate [7]. The commonly used dose quantity is the effective dose estimates based on DLP as defined in ICRP Publication 103 in relation to four common body sections; the head, chest, abdomen and pelvis [8,9]. These are based on regional dose to patients during $\mathrm{CT}$ examination. This dose parameter are used to estimates and compare dose due to different modalities.

A number of publication has been done in an attempt to determine dose to patients undergoing $\mathrm{CT}$ examination $[10,11]$. However, all this publication are based on a specific protocol with specific machine and therefore limited in scope. In an attempt to expand the scope this study covered all the commonly used machines in Ghana with their prescribed protocols to determine the absorbed dose to patients. Some of the available publication include estimation of absorbed dose to patients in Ghana by George et al [12] and estimation of patient organ doses from CT examinations in Tanzania by Justin E. Ngaile1 and Peter K. Msaki [11]. All this were Phantom studies with specific protocol and parameters. They were all studies done to estimate absorbed dose to patients undergoing $\mathrm{CT}$ examination.

In addition, to determine the tradeoff between the selected parameters and the radiation dose to produce image quality adequate enough to answer all the clinical questions. In clinical environment image quality are properly appreciated by estimating the signal to noise ratio (SNR) of the image. That is, by estimating the difference in signal intensity between the area of interest and the background

\section{Basic Principles}

The CTDI is defined as:

$$
C T D I=\frac{1}{N . T} \int_{\infty}^{\infty} D(Z) d x
$$

Where;

$\mathrm{D}(\mathrm{z})=$ the radiation dose profile along the $\mathrm{z}$-axis,

$\mathrm{N}=$ the number of tomographic sections imaged in a single axial scan.

$\mathrm{T}=$ the width of the tomographic section along the $\mathrm{z}-$ axis imaged by one data Channel

Also, 


$$
C T D I_{V O L}=C T D I_{W} / P I T C H
$$

In multislice CT (MSCT), the slice thickness is determined by the number of detectors and the widths of the detectors.

In spiral CT there is an additional factor called the CT pitch factor. It is defined as the table movement per gantry rotation:

Mathematically as

$$
\text { PITCH }=\frac{\Delta d}{N . T}
$$

Where $\Delta \mathrm{d}$ is the distance in $\mathrm{mm}$ that the couch moves between consecutive serial scans or per $360^{\circ}$ rotation in helical scanning.

$\mathrm{N}$ is the number of detectors and $\mathrm{T}$ is the detector thickness in mm [14] (IEC 2003).

The dose-length product, DLP, includes the irradiated volume and represents the overall exposure for an examination and is calculated as:

$$
D L P=C T D I_{V O L} * L
$$

Where $\mathrm{L}$ is the scan length of an examination.

\section{METHODS AND MATERIAL}

\section{A. Materials}

The materials used are shown in Figure 1-4

Table 1. Specifications of CT scanners

\begin{tabular}{|l|l|}
\hline Manufacturer & Scanner modellscan mode \\
\hline Philips Brilliance 64 & Billiance 64, multi slice, axial and helical modes \\
\hline Siemens Emotion 16 & Emotion 16, multi slice, axial and helical modes \\
\hline GE Lightspeed VCT 64 & Lightspeed VCT 64, multi slice axial and helical modes \\
\hline Toshiba Aquilion ONE & Aquilion ONE, multi slice, axial and helical modes \\
\hline
\end{tabular}

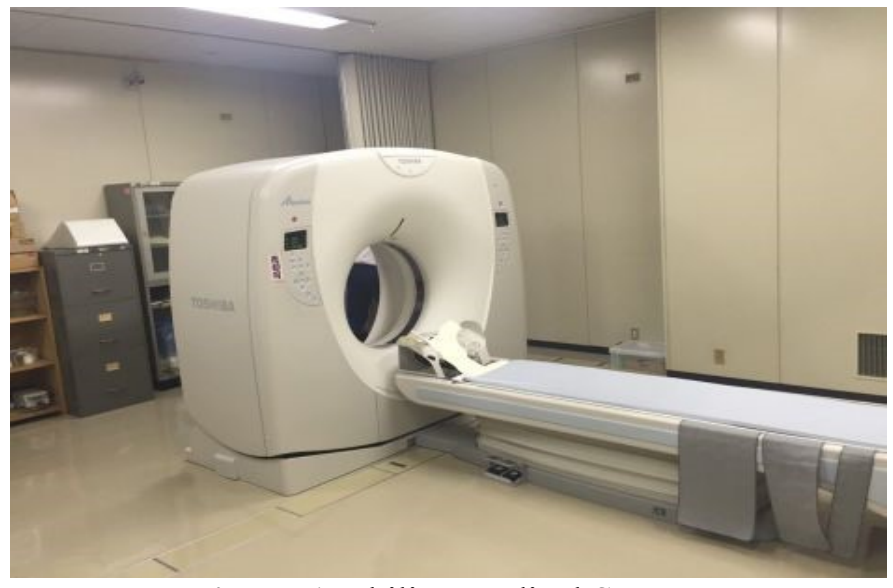

Figure 1. Philips medical System

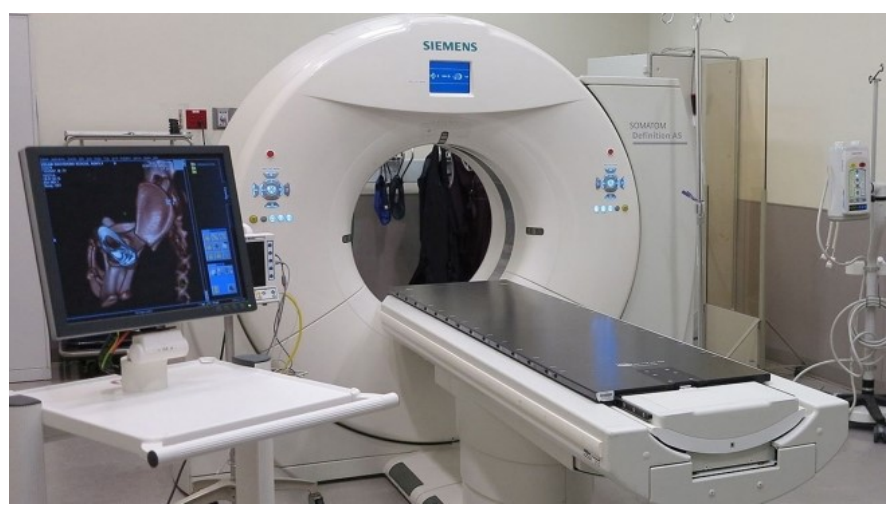

Figure 2. Siemens Medical Systems

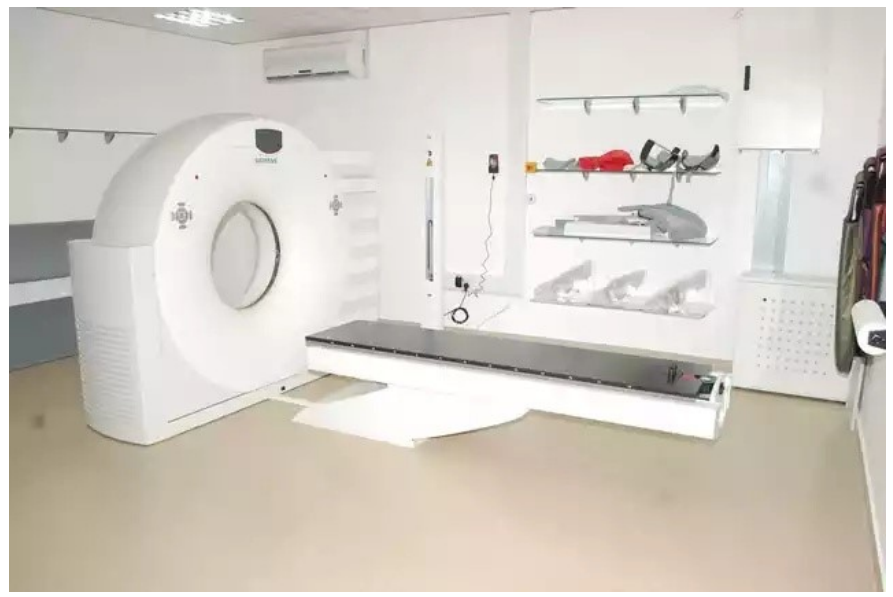

Figure 3. Toshiba Medical Systems 


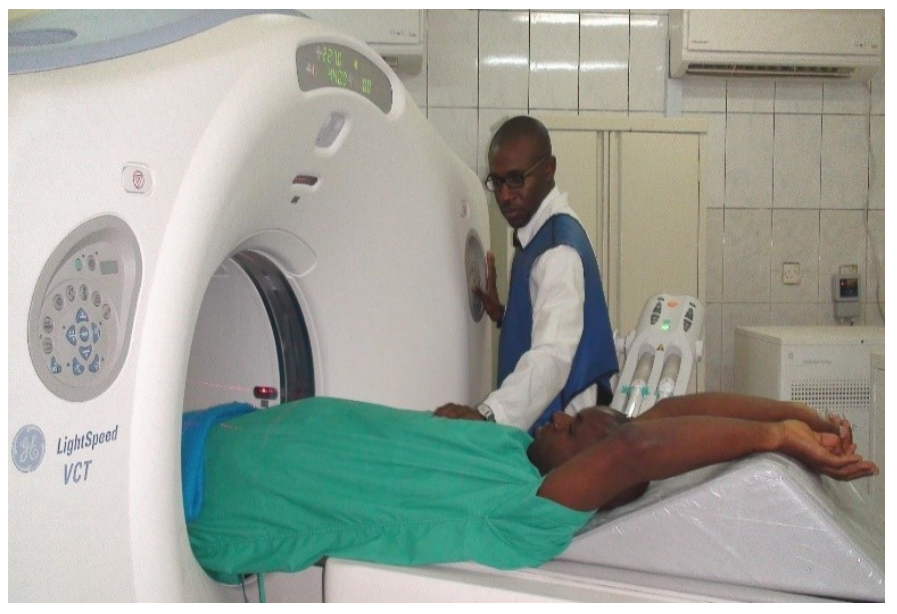

Figure 4. GE Medical Systems

\section{B. Methods}

The data used in this study were collected from four hospitals in Ghana with four different CT scanners. Detailed specifications of the scanner used at each of these hospitals are contained in the Table 1. The study reviewed $1920 \mathrm{CT}$ images from four CT units in order to determine the CTDI and DLP of patients undergoing CT examination in Ghana. Furthermore, the study uses four different CT scanners from different manufacturer; General Electric (GE), Philips, Siemens, and Toshiba. The effective dose was determined and then compared with international guidelines. Set out by the International Commission on Radiological Protection (ICRP), American College of Radiology (ACR), American Association of Physicists in Medicine (AAPM), United Kingdom (U.K.) Health Protection Agency, International Atomic Energy Agency (IAEA), and European Commission (EC)

Patient data collected during imaging from these centers in relation to the effects of patient-related parameters (e.g., age and body region in relation to a specific CT type) on regional effective dose. The selected centers and procedures in this study represented over $50 \%$ of the total CT examinations conducted in Ghana today. The collection of patient data was done with approval of ethical review of university of cape coast. An average of 600 CT examinations of head, chest, abdomen and pelvis was collected, making a total collection of over 2000 examinations.

The homogeneous volume method was used to estimate signal to noise ratio of an image by finding a homogeneous area within the image and compute the signal (process average) and the noise (standard deviation). The ratio of the mean to the standard deviation gives an estimate for the signal to noise ratio of the image. This were done together with the background process average and standard deviation outside the area of interest and the signal to noise ratio estimated. This was estimated as:

SNR was estimated using the mathematically express as:

$$
S N R=\frac{\mu(\text { Signal on organ })-\mu(\text { signal on background })}{\text { Noise }(\sigma)}
$$

\section{RESULTS AND DISCUSSION}

\section{A. Results}

The results for each CT scans under typical operation conditions for each scanner are presented in Table 3.1 and analyzed by scanner type, age and body regional specifics. The results of this are presented in four tables from Table 1 to Table 4.

Table 2. Head scanning and dose parameters

\begin{tabular}{|l|l|l|l|}
\hline Scanner (Manufacturers) & CTDIvol (mGy) & DLP mGy-cm & Effective dose mSv \\
\hline Philips brilliance 64 & 6.6 & 224.49 & 0.426531 \\
\hline Siemen Emotion 16 & 5.9 & 176.9 & 0.33611 \\
\hline GE Lightspeed VCT 64 & 7.7 & 250.00 & 0.475 \\
\hline Toshiba Aquilion one & 7.2 & 239.96 & 0.455924 \\
\hline
\end{tabular}

Table 3. Chest scanning and dose parameters

\begin{tabular}{|l|l|l|l|}
\hline Scanner (Manufacturers) & CTDIvol $(\mathrm{mGy})$ & DLP mGy-cm & Effective dose mSv \\
\hline Philips brilliance 64 & 13.1 & 773.28 & 11.21256 \\
\hline Siemen Emotion 16 & 12.5 & 708.71 & 10.2763 \\
\hline GE Lightspeed VCT 64 & 14.2 & 803.11 & 11.6451 \\
\hline Toshiba Aquilion one & 13.4 & 781.1 & 11.32595 \\
\hline
\end{tabular}

Table 4. Abdomen scanning and dose parameters

\begin{tabular}{|l|l|l|l|}
\hline Scanner (Manufacturers) & CTDIvol (mGy) & DLP mGy-cm & Effective dose mSv \\
\hline Philips brilliance 64 & 14.8 & 702.96 & 10.75529 \\
\hline Siemen Emotion 16 & 14.4 & 562.86 & 8.611758 \\
\hline GE Lightspeed VCT 64 & 16.8 & 828.8 & 12.68064 \\
\hline Toshiba Aquilion one & 15.2 & 725.12 & 11.09434 \\
\hline
\end{tabular}


Table 5. Pelvis scanning and dose parameters

\begin{tabular}{|l|l|l|l|}
\hline Scanner (Manufacturers) & CTDIvol (mGy) & DLP mGy-cm & Effective dose mSv \\
\hline Philips brilliance 64 & 12.9 & 384.79 & 4.963791 \\
\hline Siemen Emotion 16 & 12.0 & 357.6 & 4.61304 \\
\hline GE Lightspeed VCT 64 & 14.5 & 490.00 & 6.321 \\
\hline Toshiba Aquilion one & 13.5 & 436.5 & 5.63085 \\
\hline
\end{tabular}

\section{B. Discussions}

Two reference dose parameters are commonly used for CT dose estimate in order to promote the use of good technique include; CTDI and DLP. CTDI is measured as weighted CTDI $\left(\mathrm{CTDI}_{\mathrm{w}}\right)$ or volume CTDI $\left(\mathrm{CTDI}_{\mathrm{vol}}\right)$. Both CTDI and DLP parameters are used for head and body CT dosimetry which is an appropriate to the type of examination. In addition it provides control on the selection of exposure setting, such as mAs and voltage. DLP also provide control on the volume of irradiated area and the overall total exposure for an examination. They can be used for both helical scanning and serial scanning of head and body examination.

The mean values of scanning parameters $80-120 \mathrm{kV}$ peak voltage, 0.5 to 1.0 pitch factor, 0.33 to $1 \mathrm{~mm}$ slice thickness and $125-200 \mathrm{mAs}$ conducted in each of the CT examinations of head, chest, abdomen and pelvis. The results of the analysis are presented in Table 2 to Table 5. From the tables, it is observed that CT scanning protocols used for a $\mathrm{CT}$ examination per scanner per hospital were highly standardized, similar to what has been experienced in other studies. For example, children and adults patient were examined with different exposure settings, such as pitch factor, slice thickness, $\mathrm{kV}$ and $\mathrm{mA}$. This was in clinical practice, where all the protocols were observed.

It was observed that the scanning protocol in various centers in terms of patient dose increases with decreasing body size. The standardized principles in scanning protocols in all centers were uniform across hospitals. The $\mathrm{kV}$ was the same between 80 to $120 \mathrm{kV}$ in all hospitals and the mAs product variation ranging from $100 \mathrm{mAs}$ to $250 \mathrm{mAs}$ for pelvis, $60 \mathrm{mAs}$ to 350 $\mathrm{mAs}$ for abdomen $60 \mathrm{mAs}$ to $350 \mathrm{mAs}$ for chest and 60 $\mathrm{mAs}$ to $360 \mathrm{mAs}$ for head. In general, the lowest value was consistently used for all the hospitals, while the highest values varied from one hospital to another. This were all base on the scanning protocol by the various hospitals. The variation of mAs per given examination would be expected because of the difference in focus to isocenter distance among scanners.

Of interest in this study is the determination of regional body doses. However, as described, this quantity was determined after recording the $\mathrm{CTDI}_{\mathrm{vol}}$ on the console after each scan. The corresponding DLP values were also estimated and used to estimate effective dose using ICRP publication 103.

It is interesting to observe that different models of scanners have such large deviations of CTDI as in the table above and hence, regional body dose. All the scanning protocols of all the scanners show that the head produce minimum scanning and dose parameters which varies from 5.9 to 7.7 for $\mathrm{CTDI}_{\mathrm{VOL}}, 176.9$ to $250 \mathrm{mGy}$ for DLP and 0.33 to $0.47 \mathrm{mSv}$ for effective dose as shown in Table 4.1. The abdominal scanning parameters had the highest scanning and dose estimates. From a minimum of 14.4 to $16.8 \mathrm{mGy}$ for $\mathrm{CTDI}_{\mathrm{VOL}}, 562.86$ to $828.8 \mathrm{mGy}$ of DLP and 8.61 to $12.68 \mathrm{mSv}$ for effective dose. The relatively small values shown for Siemens Emotion are probably a function of their short focus-toaxial distance. As this function may reflect in the three other scanner whose focus-to-axial distance increases in the order; Philip, Toshiba and GE scanning protocols.

The increases may be due to the fact that radiation intensity varies as the inverse of the squared distance between the source of the radiation and the object (patient) [3, 4]. As a result, if all other scanning parameters are held constant, the scanner with the shorter distance between the X-ray tube focal spot and the isocenter of the gantry aperture can produce more radiation exposure than the long geometry scanner. On the other hand, the variation of source-to-detector distance among scanners might affect the image quality. This is due to the fact that image noise in CT is known to be inversely proportional to the square root of the number of photons received by the detector, whereas the number of photons (dose) is inversely proportional to the squared distance between the source of the radiation and the detector. As a result, if all other scanning parameters are held constant, the scanner with the long distance between source and detector can have higher image noise than the short geometry scanner $[4,5]$.

To establish a tradeoff between image quality and corresponding dose for patients' dose optimisation procedure, a simple numerical method was used to 
calculate the SNR ratio of CT image. That is, the ratio of the Signal (process average) over the Noise (standard deviation) were estimated. The dose optimisation process was done by plotting a graph of SNR as against effective or renal dose. Three difference abdominal tissues (lungs, kidney and spine) in the abdominal image were analyzed to establish the relationship between SNR and dose parameters detail summary of these parameters. The signal to noise ratio (SNR) as against the dose parameters on the various tissues shows the spine which is a hard tissue received higher signals strength followed by the soft kidney tissues and then the air filled sponge lungs tissues. Conversely, the spine has a smaller numerical value, followed by the kidney and then the lungs, this implied that as the signal increases due to an increases in tissues density, there is a corresponding reduction in the level of noise hence this increases image quality.

\section{Comparison with International Guideline}

The over average values of the effective dose values were within the accepted values of the UK, ICRP, AAPM, ACR and the EC recommendations values as shown in Table 6 and 7 respectively.

In conclusion the values were mostly comparable to and slightly higher than the values of body regional dose reported in literature for the EC, IAEA, ICRP and that of ACR Guidelines as shown in Table 6 .

Table 6. International guidelines and recommendation

\begin{tabular}{|l|l|l|l|l|}
\hline Body region & CDTI/DLPICRP & CDTI/DLPEC & CDTI/DLPACR & CDTI/DLPAAPM \\
\hline Head & $50 / 1050$ & $60 / 1050$ & $55.8 / 837$ & $55.0 / 825$ \\
\hline Chest & $30 / 650$ & $30 / 650$ & $25 / 1000$ & $12.0 / 480$ \\
\hline Abdomen & $20 / 780$ & $35 / 780$ & $25 / 500$ & $19.1 / 382$ \\
\hline Pelvis & $20 / 780$ & $40 / 780$ & $15 / 600$ & $19.1 / 764$ \\
\hline
\end{tabular}

Table 7. International guidelines and standards

\begin{tabular}{|l|c|c|c|c|}
\hline \multicolumn{1}{|c|}{ Examination } & Mean values & Japan & UK & EC \\
\hline Head CT & & & & \\
\hline CTDI $_{w}(m G y)$ & 39 & 47 & 66 & 60 \\
\hline DLP $(m G y-c m)$ & 544 & 527 & 787 & 1050 \\
\hline Chest CT & & & & \\
\hline CTDI $(m G y)$ & 9.3 & 9.5 & 17 & 30 \\
\hline DLP $(m G y-c m)$ & 348 & 447 & 488 & 650 \\
\hline Abdominal cT & & & & \\
\hline CTDI $(m G y)$ & 10.4 & 10.9 & 19.0 & 35 \\
\hline DLP $(m G y-c m)$ & 549 & 696 & 472 & 780 \\
\hline
\end{tabular}

\section{CONCLUSION}

Assessment of radiation dose to regional body section of patients undergoing CT examinations in Ghana was investigated. In this study. The variations of radiation dose to head, chest, abdomen and pelvis were observed. Different scanning protocols used among different scanners at different hospitals and variation in equipment design among manufacturers and models were responsible for these variations. The mean effective and other dose parameters in this study were mostly comparable to and slightly higher than reported values from EC, IAEA, ICRP and ACR guidelines. The main contributor for this difference was the use of a larger scan length. The large observed variations of regional body doses among hospitals and relatively high doses at various hospitals call for the need to optimize CT scanning protocols. This can be achieved through optimal selection of scanning parameters based on indication of study, body region of interest being scanned, and patient size. In addition, further studies should be done to vary the various protocols and fully implement ICRP publication 103.

\section{RECOMMENDATION}

$\checkmark$ In view of the observed causes of regional body dose variations and similar experience observed elsewhere, further studies on the optimization of doses to patients undergoing CT examinations in Ghana are needed. There are a number of observed parameters that greatly need optimization,

$\checkmark$ In order to achieve the above optimization strategies, there is a great demand to educate CT personnel on the effects of scan parameter settings on radiation dose to patients and image quality required for accurate diagnosis

$\checkmark$ The first is the minimization of the number of slices (hence scan length) as much as possible, without missing any vital anatomical region. Several studies have recommended that with the reduction of irradiation volume depending on body region being scanned, radiation dose to patients can be significantly reduced.

The second is minimization of tube current $(\mathrm{mA})$ based on indication of study. Some studies have already revealed that adjustment of $\mathrm{mA}$ based on indication of study dose to patient can be reduced to 
$50 \%$ without significantly affecting the image quality.

$\checkmark$ The third approach is through modulation of exposure parameters (i.e., $\mathrm{kV}, \mathrm{mA}$, exposure time, and slice thickness) based on patient size and age, while maintaining a constant contrast to image-tonoise ratio. Some studies have demonstrated that by adjusting the exposure parameters based on patient age, weight, or transverse diameter of the body part imaged, patient radiation doses can be reduced significantly.

$\checkmark$ Another possible method is through use of contrast media only to optimize diagnostic yield. As mentioned earlier, use of contrast media by some of the hospitals was done as a routine procedure without clinical justification of using it.

\section{ACKNOWLEDGEMENTS}

My sincere thanks goes to the entire staff of the Medical Radiation Physics Centre, Radiological and Medical Sciences Research Institute of Ghana Atomic Energy Commission, Department of Radiology, Korle-Bu Teaching Hospital, Tamale Teaching Hospital, Komfo Anokye Teaching Hospital, Cocoa clinic and 37 Military Hospital, for their support and cooperation.

Finally, I take this opportunity to express my sincere and heartfelt appreciation to University of Cape Coast, for the opportunity granted me during the course of this research work as part of my $\mathrm{PhD}$ study, especially Dr. Alfred Owusu whose letter of introduction enable me easy access to the various facilities for data collection.

\section{REFERENCES}

[1]. European Commission. European guidelines on quality criteria for computed tomography EUR 16262 EN, Luxemburg. Office for Official Publications of the European Communities; 1999.

[2]. United Nations Scientific Committee on Effects of Radiation Atomic Radiation (UNSCEAR). Sources and effects of ionizing radiation report to the general assembly. New York: United Nations; 2000 .
[3]. International Atomic Energy Agency (IAEA). Protection of patients in diagnostic and intervention radiology, nuclear medicine and radiotherapy. Proceeding of International Conference, Malaga, Spain, 26-30 March 2001; Vienna.

[4]. International Commission on Radiological Protection (ICRP). Managing patient dose in computed tomography annals of ICRP publication 87; 30(4). Oxford: Pergamon Press; 2000.

[5]. Hidajat $\mathrm{N}$, Maurer $\mathrm{J}$, Schroder RJ, et al. Relationships between physical dose quantities and patient dose in CT. Br J Radiol. 1999; 72:556561.

[6]. Dr Henry Knipe and Dr Usman Bashir et al, CT dose index, UDM medical network, Radiopeadia.org

[7]. Hopper KD, Neumann JD, King SH, Kunselman AR. Radioprotection to the eye during CT scanning. Am J Neuroradiology. 2001; 22:11941198.

[8]. Hopper KD, King SH, Lobell ME, TenHave TR, Weaver JS. The breast: In-plane X-ray protection during diagnostic thoracic CT-shielding with bismuth garments. Radiology 1997; 205:853-858.

[9]. Geleijns J, Van Unnik JG, Zoetelief J, Zweers D, Broerse JJ. Comparison of two methods for assessing patient dose from computed tomography. Br J Radiol. 1994; 67:360-365.

[10]. Shrimpton PC, Jones DG, Hillier MC, et al. Survey of CT practice in the UK. Part 2: Dosimetric aspects. Chilton, NRPB-R249. London: HMSO; 1991

[11]. Thomas JEM, Tingey DRC. Radiation doses from computed tomography in Australia. Australian Radiation Laboratory Report, ARL/TR123; 1997.

[12]. Mayo JR, Hartman TE, Lee KS, Primack SL, Vedal S, Muller NL. CT of the chest: Minimal tube current required for good image quality with the least radiation dose. Am J Roentgenol. 1995; 164:603-607. 